\title{
Silica-Shielded Ga-ZnS Metal-Semiconductor Nanowire Heterojunctions
}

\author{
J. Q. Hu, ${ }^{*}$ Y. Bando, ** J. H. Zhan, ${ }^{* *}$ and D. Golberg**
}

* International Center for Young Scientists, National Institute for Materials Science, Namiki 1-1, Tsukuba, Ibaraki 305-0044, Japan

** Advanced Materials and Nanomaterials Laboratories, National Institute for Materials Science, Namiki 1-1, Tsukuba, Ibaraki 305-0044, Japan

One-dimensional (1D) nanostructures possessing heterojunctions are of particular interest with respect to potential applications in nanoelectronics and nanophotonics [1]. For example, sophisticated light-emitting diodes (LEDs) [2] and diode logic [3-4] devices have been realized while crossing $\mathrm{p}$ - and n-type nanowires or using lithography assisted selection of distinct $\mathrm{p}$ - and ntype regions within a nanotube [5]. However, compared with a significant progress in nanowire and nanotube preparation in many homogeneous systems, the desired heterojunction formation with well-defined interfaces within 1D nanostructures has been lingering far behind. Most recently, we have reported on the fabrication of new indium-silicon (In-Si) end-to-end nanowire contacts, i.e. metal-semiconductor heterojunctions [6]. Here, we report on the fabrication of Ga-ZnS metalsemiconductor nanowire heterojunctions uniformly sheathed with very thin silica nanotube. The nanostructures are prepared through thermal evaporation of $\mathrm{SiO}, \mathrm{Ga}_{2} \mathrm{O}_{3}$, and $\mathrm{ZnS}$ powder precursors followed by desired thermo-chemical reactions. Most of the $\mathrm{Ga}-\mathrm{ZnS}$ nanowire heterojunctions have diameters of $\sim 150-250 \mathrm{~nm}$ (a few of them have diameters of $\sim 80-120 \mathrm{~nm}$ ); wall thickness of shielding silica tubes is $\sim 4-8 \mathrm{~nm}$. Some nanostructures have a single junction; the others have two or more periodically-located Ga-ZnS junctions (see Figure 1). An interface between Ga and $\mathrm{ZnS}$ domains is found to be particularly sensitive to the electron beam irradiation within a transmission electron microscope (see Figure 2). This may open prospects for the design of a unique electron beam - and temperature-driven switch and/or sensor within an electronic device.

References

[1] S. M. Sze, Physics of Semiconductor Devices, Wiley-Interscience, New York, 1981.

[2] X. F. Duan, et al., Nature 409 (2001) 66.

[3] A. Bachtold, et al., Science 294 (2001) 1317.

[4] V. Derycke, et al., Nano Lett. 1 (2001) 453.

[5] Y. Huang, et al., Science 291 (2001) 630.

[6] J. H. Zhan, et al., Angew. Chem. Int. Ed. 44 (2005) 2140.

[7] This work was performed through the Special Coordination Funds for Promoting Science and Technology from the Ministry of Education, Culture, Sport, Science, and Technology of the Japanese Government. 

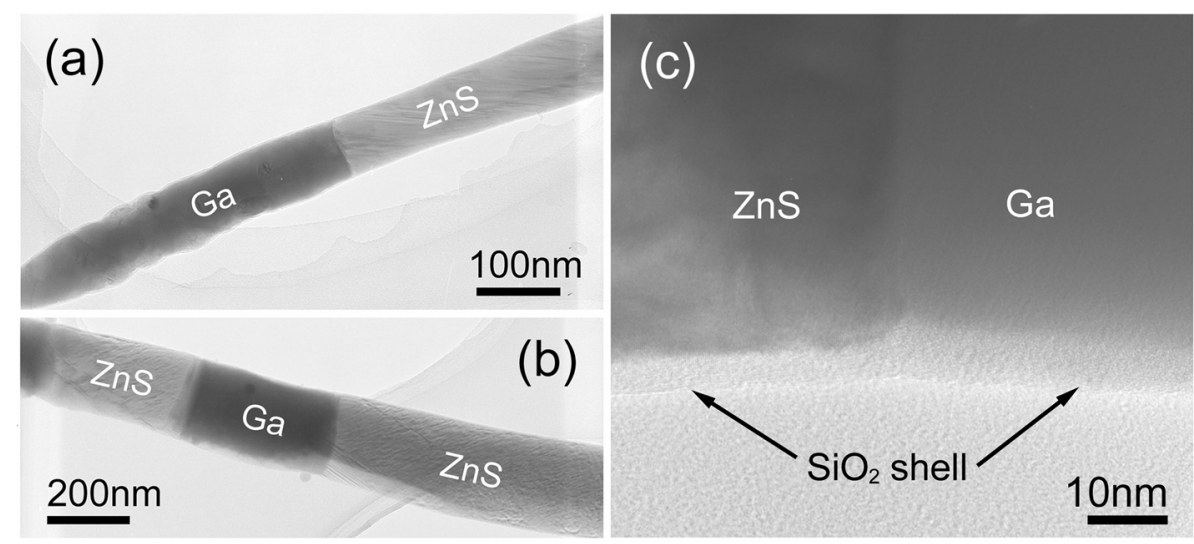

FIG. 1. (a), (b) TEM images shows the Ga-ZnS nanowire heterojunctions having one and two junctions along the length, respectively. (c) High-magnification TEM image shows the Ga-ZnS nanowire junction is homogeneously sheathed with very thin $\mathrm{SiO}_{2}$ tubular layer.

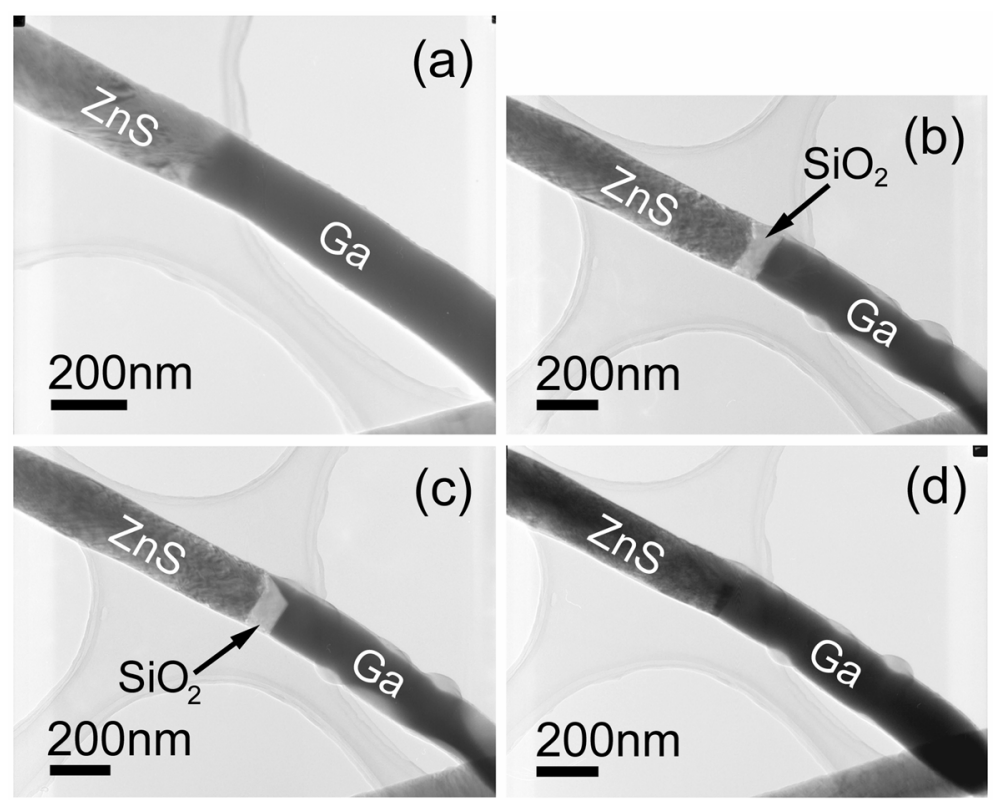

FIG. 2. Consecutive TEM images depicting in-situ EBI on a junction area causing the move of a Ga nanowire apart from an original Ga-ZnS junction: (a) before irradiation, (b) cutting junction using a convergent electron-beam (with a size of $\sim 200 \mathrm{~nm}$ ) focused on the junction area, (c) a junction recovery process when the beam is kept away (but still is close to the junction area), and (d) a complete recovery when the beam is placed far away from the junction. 